Internationale Zeitschrift für

\section{Bauinstandsetzen}

10. Jahrgang, Heft 4, 2004
International Journal for

Restoration

Vol. 10, No. 4, 2004

\title{
Inhalt / Contents
}

Editorial

E. Giuriani and G. Metelli

The Role of Sapwood Plasticity in the Delamination Phenomenon of Repaired Timber Beams

Die Rolle der Plastizität von Splintholz beim Vorgang der Delaminierung von verstärkten Holzbalken

S. W. Massey

An Alternative Method for Correction of Data for the Effect of Drill Bit Wearing in Drilling Tests Dealing with Heterogeneous Material Eine ergänzende Methode zur Korrektur von Messdaten bezüglich des Abriebs des Bohrers bei Versuchen zur Bestimmung des Bohrwiderstands von heterogenen Werkstoffen

G. M. E. Kamh

Geologic and Geotechnical Characterization of the Sandstone for Restoration of Archaeological Sites, Fourteen Triassic Red Sandstone Quarries in Great Britain, a Case Study Geologische und geotechnische Kennzeichnung von Sandsteinarten für die Restaurierung von archäologischen Stätten, Vierzehn Sandsteinbrüche in Großbritannien mit rotem Sandstein aus dem Trias, eine Fallstudie

M. Bach, F. Feugeas, F. Farcas, E. Marie-Victoire and A. Cornet

Electrochemical Evaluation of an Organic Corrosion Inhibitor

to Prevent Decay in Stone Monuments

Elektrochemische Evaluation eines organischen Korrosionsinhibitors

von Stahl, um dem Verfall von Stein Monumenten vorzubeugen

Empfehlungen für die Praxis

Hydrophobieren von Betonoberflächen

Empfehlungen für die Applikation (vorläufige Fassung) 


\section{Aus der Praxis / Applications \\ C. Weißlein \\ Niederdruckstrahltechnik - Traditionsbruch zu Gunsten einer idealen Oberflächentechnik}

\section{Mitteilungen / Short Notes}

Neue Formmassen von Perstorp schützen öffentliche Einrichtungen

gegen die Verbreitung von Krankheiten

Wie kann Schilfsandstein konserviert werden?

Restaurierung historischer Glasmalereien: Alte Pracht in neuem Glanz

Summer_lab Dessau "Freilegungen/Excavations"; 23.8. - 10.9. 2004

EMiG-Tagung am 23. September 2004

Gebäude von heute - Ladenhüter von morgen?

\section{Firmenmitteilungen / Companies Inform}

Warum ist Blähschiefer unempfindlich gegenüber Treibmineralbildung?

Gerb gibt Broschüre und $C D$-Rom heraus:

Neue Technik zur Fundament-Sanierung

\section{Neuerscheinungen /New Books}

\section{WTA/Info}

